\title{
INTERFEROMETRIC POINT TARGET ANALYSIS (IPTA) FOR LANDSLIDE MONITORING
}

\author{
M.Yarmohammad touski ${ }^{1}{ }^{*}$, M. Veiskarami ${ }^{1}$, M. Dehghani ${ }^{1}$ \\ ${ }^{1}$ Dept. of Civil and Environmental Engineering, School of Engineering, Shiraz University, Shiraz, Iran \\ yarmohammadi.milad@yahoo.com - mveiskarami@shirazu.ac.ir - maryamdehghani@shirazu.ac.ir
}

KEY WORDS: Landslide, SAR Interferometry, IPTA, Persistent Scatterer

\begin{abstract}
:
By the advent of Persistent Scatterer Interferometry (PSI) time-series analysis, this technique has demonstrated high performance in producing accurate measurements of ground displacements. However, due to several limitations such as high deformation rate, lack of man-made features and rough topographic characteristic, the efficiency of the PSI significantly decreases. The main goal of this paper is to illustrate the potential of one of the PSI methods namely the Interferometric Point Target Analysis (IPTA) to measure the deformation caused by landslide. The landslide occurs in an area lacking man-made features with rough topography. To this end, 28 Sentinel-1A SLC images spanning from October 142014 to October 27 2016, were used to generate single-master interferograms. The PS pixels were identified using amplitude dispersion and spectral diversity criteria. The PS pixels were unwrapped considering a linear model for the deformation behavior in an iterative manner. The residual topography and atmospheric contributions were estimated in each iteration and subtracted from the PS pixels phases. The results were finally compared to those extracted from conventional Small Baseline Subset (SBAS) time series analysis applied on the same datasets. A good agreement existed between two methods in some locations whereas unwrapping errors probably due to improper deformation model were observed in a couple of points.
\end{abstract}

\section{INTRODUCTION}

\subsection{General Instructions}

Since the early 1990s, Synthetic Aperture Radar (SAR) interferometry (InSAR) which is a combination of two or multiple SAR images has been used as an effective method for monitoring geo hazard phenomena including land subsidence, volcanic eruptions and landslides which are deforming Earth's surface. Covering a large field of study in a lower cost and time compared to the previous method such as precise leveling can be viewed as the beauty of this technique.

InSAR, however, fails to measure the deformation provided that the area is covered by significant amount of vegetation, rough topography and remarkable atmospheric effects which result in decorrelation effects. In the late 1990s, it was found that there are specific targets on the Earth's surface which seems to have little variation in their backscattering behavior. These targets are called Persistent Scatterers (PS). The deformation can then be monitored on these PS pixels using the principles of InSAR. Consequently, several PS Interferometry (PSI) algorithms have been developed (Dehghani et al., 2013).

The main objective of this paper is to illustrate the potential of one of the PSI technique called Interferometric Point Target Analysis (IPTA) to monitor Kahroud landslide. Landslide is one of the hazards that causes serious damage to man-made structures and nature. Its movement begins when slope failure happen in response to the increased ratio of destabilizing shear stress to resisting shear strength. Studies suggest that most of landslides have spatial and temporal complex behavior (Hu et al., 2016; Tong \& Schmidt, 2016).

Kahroud landslide located in north of Iran is an active one with a complex behavior in both space and time. It is, however, subject to the low degree of decorrelation so as the conventional InSAR can also lead to the reliable results. Therefore, in order to evaluate the performance of IPTA in monitoring Kahroud landslide, the results obtained from this method were compared to those achieved by conventional INSAR.

\subsection{Data sets and case study}

The study area located in the upstream of Kahroud city is show in Figure 1. The area located in the central Alborz Mountains (Iran) in the Haraz valley is characterized by rough topography. The deformation caused by Kahroud landslide is considered as a major threat to the Haraz route and it's around infrastructures. Due to the rough topography in the study area, a little difference in incidence and squint angles in slave and master images will result in a significant decorrelation effect. Moreover, the area lacks man-made features.

In the past, studies have been conducted using various interferometric techniques and global positioning system on this area (Dehghani, 2016; Peyret et al., 2008; Tavakkoli \& Dehghani, 2015). These studies are basically based on conventional interferometry or combined method of conventional and PSI. As the potential of IPTA has not been widely evaluated for monitoring landslides, IPTA is applied. In order to study the spatial and temporal behaviour of Kahroud landslide, 28 ascending images of sentinel-1A from track 130 and frame 111 acquired between October 142014 and October 27 2016 were used for the time series analysis. Two different approaches based on InSAR, i.e. Small Baseline Subset (SBAS) time series analysis and IPTA were employed and the corresponding results were compared. Section 2 gives a brief description on both methods. The results are presented in Section 3. Section 4 is devoted to some concluding remarks.

\footnotetext{
* Corresponding author
} 


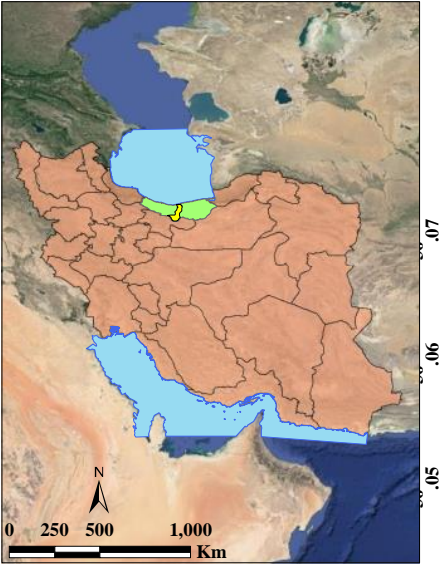

(a)

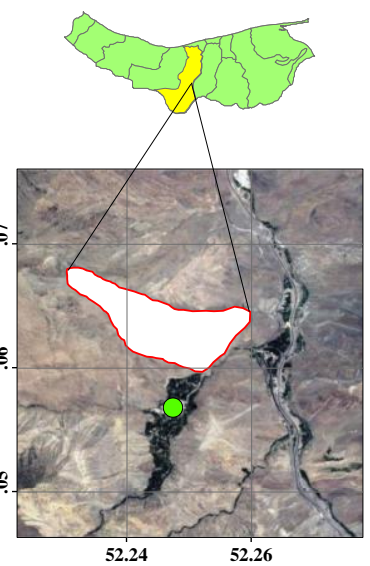

(b)
Figure 1. (a) Location of the Mazandaran province in Iran superimposed on the Google-earth image, (b) Kahroud landslide boundary depicted by red polygon. The village of Kahroud is presented by a green circle.

\section{METHODOLOGY}

The phase difference of two SAR images, known as interferometric phase, contains different components as follows:

$$
\varphi_{\text {unw }}=\varphi_{\text {curv }}+\varphi_{\text {topo }}+\varphi_{\text {def }}+\varphi_{\text {atm }}+\varphi_{\text {noise }}
$$

where $\varphi_{\text {unw }}$ is the unwrapped interferometric phase, $\varphi_{\text {curv }}$ is the phase component due to the earth curvature, $\varphi_{\text {topo }}$ is the topographic contribution to the interferometric phase, $\varphi_{\text {def }}$ is the phase component due to the deformation, $\varphi_{a t m}$ is the atmospheric phase and $\varphi_{\text {noise }}$ is the noise component corresponding to the decorrelation effects (Werner et al., 2004). In order to estimate the deformation, other components are estimated and subtracted from the interferometric phase, hereafter is called the differential interferogram. Using a significant number of differential interferograms, we are able to generate the deformation time series used for investigating the temporal and spatial behavior of the landslide.

\subsection{Conventional Interferometry (SBAS Time series)}

One approach for time series analysis is the Small Baseline Subset (SBAS) in which the processed interferograms are characterized by small spatial and temporal baselines (Berardino et al., 2002; Dehghani et al., 2009). In this study, 80 smallbaseline interferograms were generated from 28 Sentinel-1A Single Look Complex (SLC) images using the GAMMA software according to Figure 2. The spatial and temporal baseline information of the SAR data is shown in Figure 2. Sentinel-1A images have a short spatial and temporal baseline. In this study most interferograms have a temporal resolution of 24 days. The block diagram shown in Figure 3 presents several processing steps in SBAS time series analysis. The SRTM digital elevation model (DEM) with the spatial resolution of $30 \mathrm{~m}$ was used to remove the topographic component in the interferometry process. The processed interferograms were flattened by subtracting a plane fitted to the area away from the landslide. Moreover, they are calibrated according to an area outside the landslide as a spatial reference. In the time series analysis, the deformation at each image acquisition were estimated by interferograms inversion using the method of Least Squares considering the first acquisition date as a time reference. In addition, a smoothing constraint is incorporated into the inversion problem in order to decrease the atmospheric artifacts, noise and unwrapping errors (e.g. Tavakkoli \& Dehghani, 2015). It should be noted that the study area is subject to low degree of decorrelation and the processed interferograms are coherent enough to be correctly unwrapped. Therefore, the results obtained from the SBAS, is reliable enough for further comparisons.

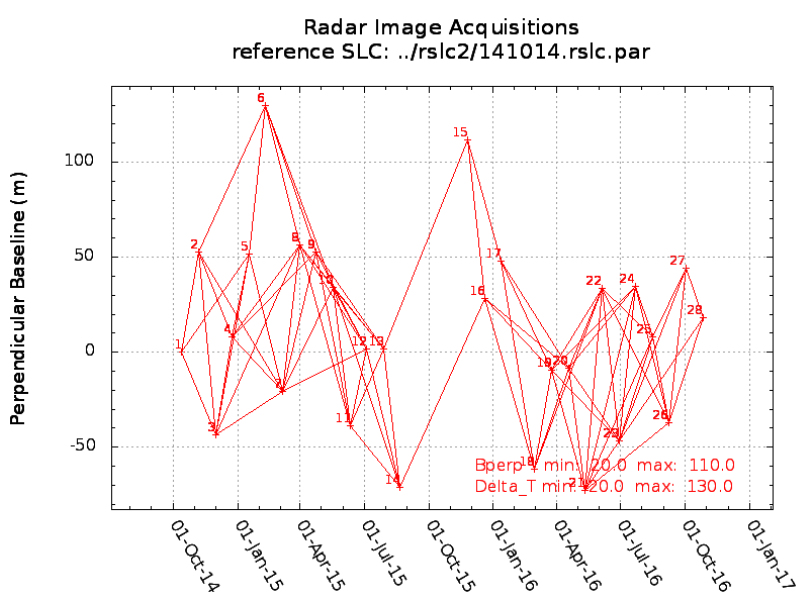

Figure 2. Interferometric pairs selected based on a stack of 28 Sentinel-1A images, with constraints on perpendicular baseline of 20 to $110 \mathrm{~m}$ and time interval of 20 to 130 days. The red lines indicate the set of SAR pairs used to form interferograms.

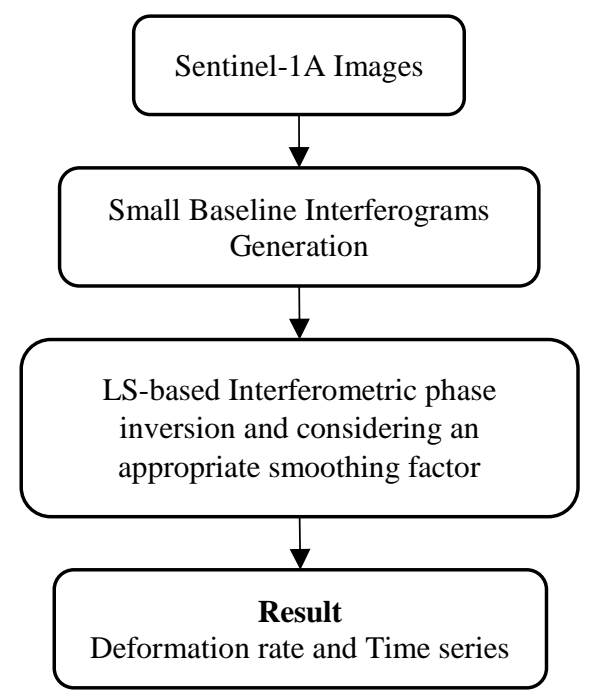

Figure 3. Overall processing steps in SBAS analysis.

\subsection{Interferometric Point Target Analysis (IPTA)}

The main objective of this paper is, however, to evaluate the performance of IPTA for monitoring a complex landslide in an area lacking man-made structures. In this method, 28 sentinel-1A images were used to generate 27 single-master interferograms in relation to the master image (December 20, 2015) is shown in Figure 4. 


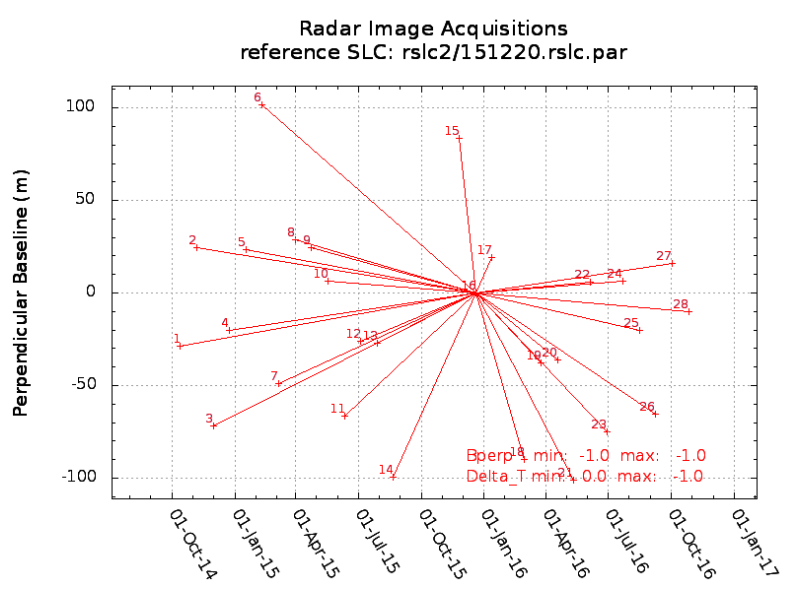

Figure 4. Interferometric pairs in relation to an image December $20,2015$.

The processing method included Sentinel-1A (SLC) image coregistration to a single master image. The PS pixels were identified using both amplitude dispersion and spectral diversity and then generate the stack of interferograms for PS candidate list. After identification of the PS candidates, assuming a linear deformation model, the PS pixels were unwrapped using appropriate ranges for deformation rate and residual topographic effects using regression in the complex domain. An example is shown in Figure 5. This regression analysis is performed for all selected points with respect to a point as spatial reference. The results of this analysis include: linear deformation rates, height corrections, a quality measure, residual phases, and the unwrapped interferometric phase (Werner et al., 2003). The final PS pixels were selected based on the unwrapping quality. The atmospheric phase screen (APS) was then estimated by a combination of spatial and temporal filtering of the Least Squares residuals and subtracted from the interferometric phase. The whole process was then repeated on the refined interferometric phase. Further iterations were continued until no change on the estimations was observed. It should be noted that the most complex deformation model used by the method of IPTA has a linear form. If the temporal deformation model of a PS pixel deviates from the linear form, the PS is removed from the candidate list (Werner et al., 2004). This will leads to the low density of the PS and consequently undesired unwrapping error. The overall processing of IPTA technique is implemented show in Figure 6.
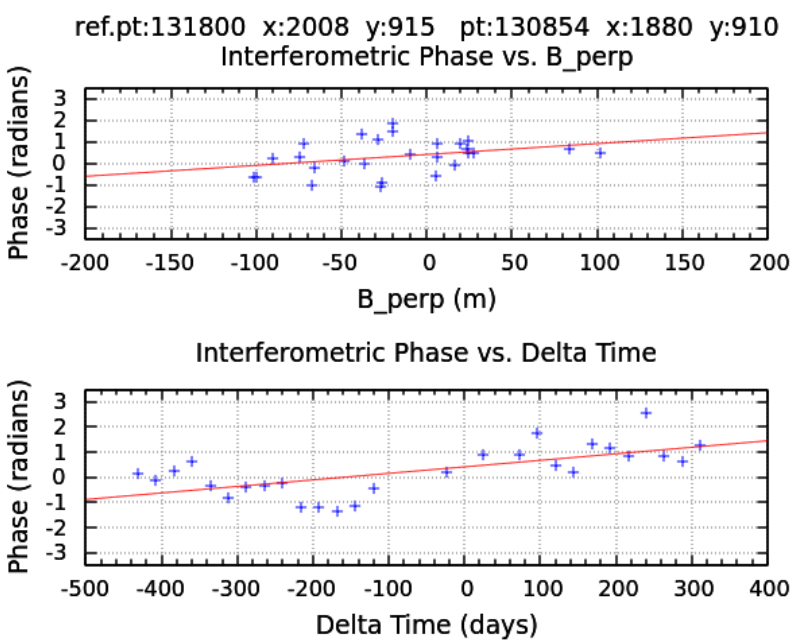

Figure 5. Fitting the two-dimensional regression model. The red line represents the regression line and the blue crossed points show the interferometric phase difference of a point and reference one in a stack of 27 Sentinel-1A interferograms. The upper plot shows the dependence of the phase to the spatial baseline corresponding to the topographic effect and the lower plot shows a temporal phase trend related to the deformation.

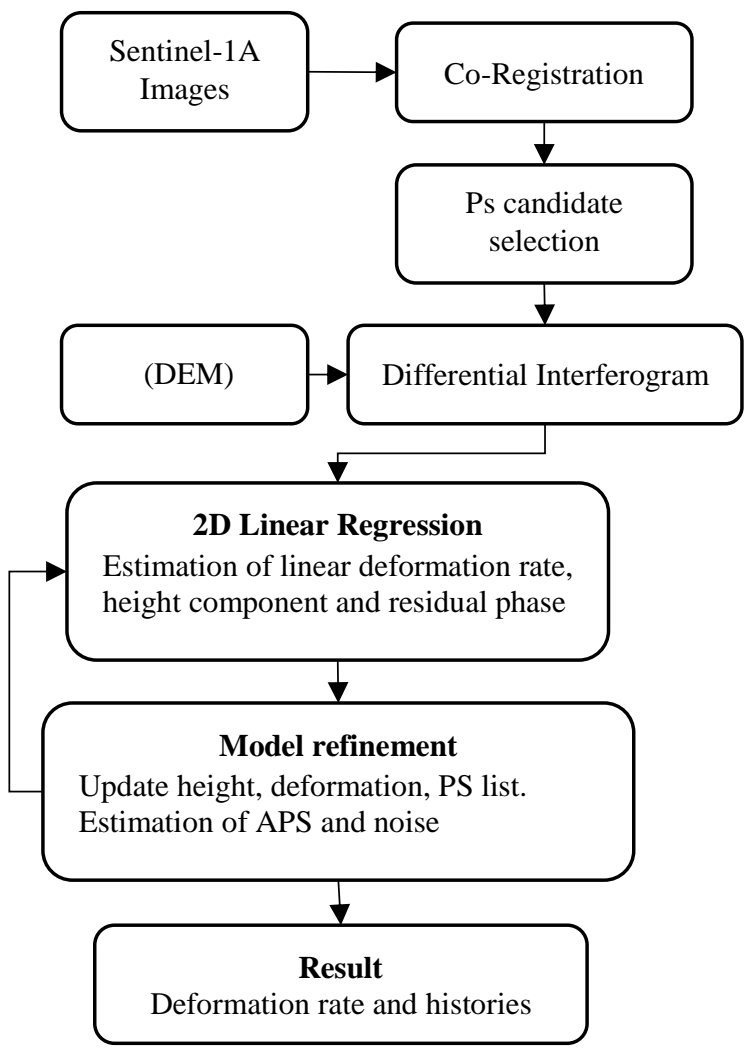

Figure 6. Interferometric point target analysis (IPTA) processing steps.

\section{RESULTS}

The deformation map obtained from IPTA and SBAS algorithm, using 28 Sentinel-1A images is shown in Figure 7 and 8. These measurements are made with respect to a reference point close to the displacement region and in a stable area.

Figure 7 shows the PS pixels detected by IPTA in Kahroud landslide whose boundary is depicted by a red polygon. The PS density within the landslide area is significantly low which might be an indication of improper deformation model. Moreover, the criteria used for PS selection are most successful in identification of targets with high amount of backscattering. The areas with natural targets rather than man-made features are mostly characterized by low backscattering.

As observed in Figure 7, the landslide is composed of two different behaviors, i.e. subsidence and uplift with the maximum deformation rate of $\sim 6 \mathrm{~cm} / \mathrm{yr}$. As mentioned in the previous section, one of the most important sources of error in IPTA is phase unwrapping. Therefore, in order to evaluate the results of this method, the SBAS time series analysis was applied. Figure 8 illustrates the deformation rate obtained from the SBAS time series analysis results. The two parts of the landslide with different behavior are again observed in the SBAS results. The maximum deformation rate is, however, different from those obtained from the method of the IPTA. 
The deformation time series at a selection of points whose locations are shown in Figure 8, were analysed for further evaluations (Figure 9 and 10). The method of IPTA and SBAS time series analysis are in a good agreement in points whose deformation time series are demonstrated in Figure 9. Some discrepancies observed in two time series might be due to different algorithms used for atmospheric effects mitigation or local unwrapping error. A systematic bias, however, is seen in the IPTA results when compared to the SBAS results in other two points (Figure 10). The IPTA results at these locations are mainly pronounced by unwrapping error.

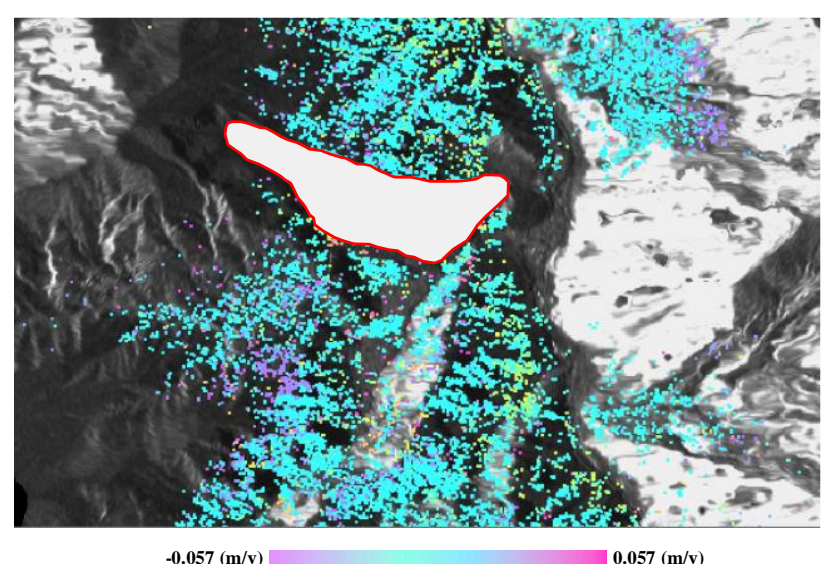

Figure 7. Persistent Scatterers (PS) selected by IPTA superimposed on Digital Elevation Model (DEM). Different colours correspond to different deformation rates.

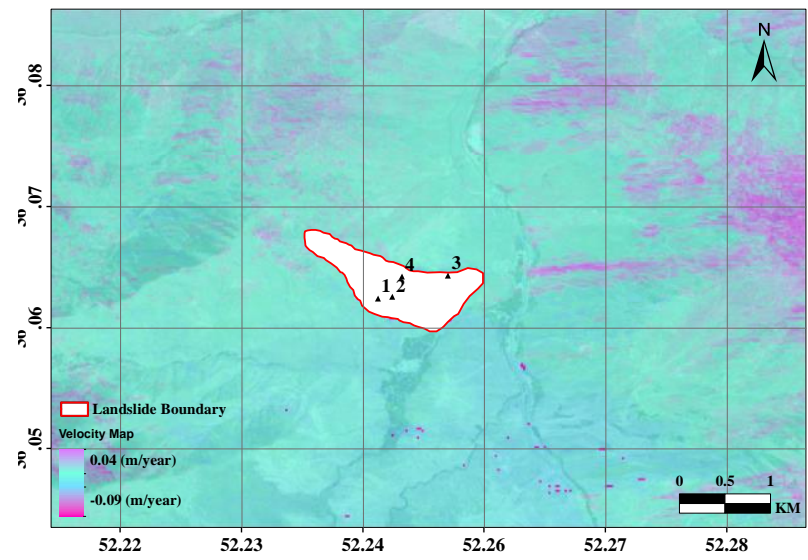

Figure 8. Deformation rate map extracted from the SBAS time series analysis results superimposed on the Google-earth image. The locations of sample points are also depicted.
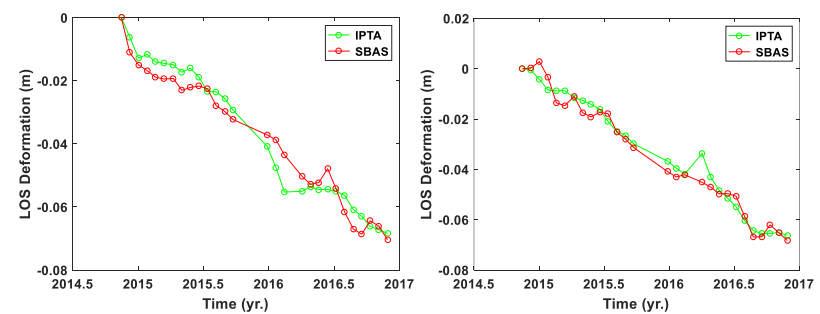

Figure 9. Deformation time series corresponding to points 3 and 4 obtained from both IPTA and SBAS.

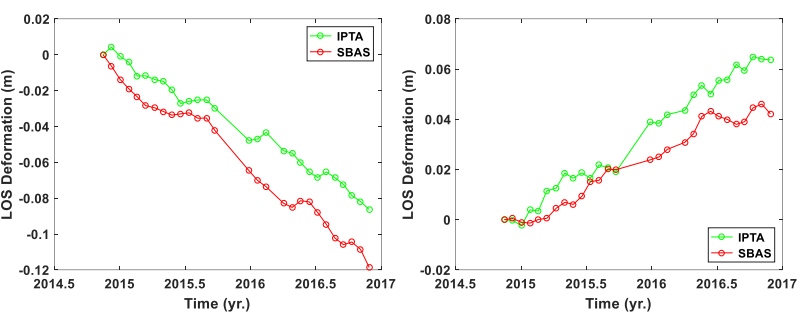

Figure 10. Deformation time series corresponding to points 1 and 2 obtained from both IPTA and SBAS.

Even more significant unwrapping errors were observed in some other locations. These locations contain a significant non-linear deformation component which IPTA fails to model. Incorporating the non-linear behavior into the deformation model would improve the quality of the results.

\section{CONCLUTION}

In this paper, two InSAR time series techniques using 28 Sentinel-1A SLC images were used in order to study the Kahroud landslide. The SBAS time series analysis was employed as a benchmark to evaluate the performance of the method of IPTA. The results showed that IPTA is able to identify the PS pixels and correctly unwrapped their phase when their deformation behavior is consistent with the pre-assumed deformation model. Any deviation of the deformation behavior from the IPTA assumption will result in the PS pixel elimination or its unwrapping error. Incorporating more complex deformation model will result in more enhancement of the IPTA performance.

\section{REFERENCE}

Berardino, P., Lanari, R., Mora, O., Mallorqui, J. J., \& Sansosti, E. (2002). A new algorithm for monitoring localized deformation phenomena based on small baseline differential SAR interferograms. IEEE International Geoscience and Remote Sensing Symposium, 2(11), 12371239. https://doi.org/10.1109/IGARSS.2002.1025900

Dehghani, M. (2016). Landslide Monitoring Using Hybrid Conventional and Persistent Scatterer Interferometry. Journal of the Indian Society of Remote Sensing, 44(4), 505-513. https://doi.org/10.1007/s12524-015-0536-3

Dehghani, M., Valadan Zoej, M. J., Entezam, I., Mansourian, A., \& Saatchi, S. (2009). InSAR monitoring of progressive land subsidence in Neyshabour, northeast Iran. Geophysical Journal International, 178(1), 47-56. https://doi.org/10.1111/j.1365-246X.2009.04135.X

Dehghani, M., Valadan Zoej, M. J., Hooper, A., Hanssen, R. F., Entezam, I., \& Saatchi, S. (2013). Hybrid conventional and Persistent Scatterer SAR interferometry for land subsidence monitoring in the Tehran Basin, Iran. ISPRS Journal of Photogrammetry and Remote Sensing, 79, 157170. https://doi.org/10.1016/j.isprsjprs.2013.02.012

Hu, X., Wang, T., Pierson, T. C., Lu, Z., Kim, J., \& Cecere, T. H. (2016). Remote Sensing of Environment Detecting seasonal landslide movement within the Cascade landslide complex ( Washington ) using time-series SAR imagery, 187, 49-61.

Peyret, M., Djamour, Y., Rizza, M., Ritz, J. F., Hurtrez, J. E., Goudarzi, M. A., ... Uri, F. (2008). MonPeyret, M., Djamour, Y., Rizza, M., Ritz, J. F., Hurtrez, J. E., 
Goudarzi, M. A., ... Uri, F. (2008). Monitoring of the large slow Kahrod landslide in Alborz mountain range (Iran) by GPS and SAR interferometry. Engineering Geology, 100(3-4), 131-141. htt. Engineering Geology, 100(3-4), 131-141. https://doi.org/10.1016/j.enggeo.2008.02.013

Tavakkoli, A., \& Dehghani, M. (2015). Kahrood monitoring using small baseline subset Synthetic Apreture Radar (SAR) interferometry. International Archives of the Photogrammetry, Remote Sensing and Spatial Information Sciences - ISPRS Archives, 40(1W5), 719723. https://doi.org/10.5194/isprsarchives-XL-1-W5-7192015

Tong, X., \& Schmidt, D. (2016). Active movement of the Cascade landslide complex in Washington from a coherence-based InSAR time series method. Remote Sensing of Environment, 186, 405-415. https://doi.org/10.1016/j.rse.2016.09.008

Werner, C., Wegmuller, U., Strozzi, T., \& Wiesmann, A. (2003). Interferometric point target analysis for deformation mapping, $\quad 00(1), \quad 4362-4364$ https://doi.org/10.1109/igarss.2003.1295516

Werner, C., Wegmuller, U., Wiesmann, A., \& Strozzi, T. (2004). Interferometric Point Target Analysis with JERS-1 L-band SAR data, (July), 4359-4361. https://doi.org/10.1109/igarss.2003.1295515 